\title{
The association of KRAS mutation with primary tumor location and survival in patients undergoing resection of colorectal cancers and synchronous liver metastases
}

\author{
Niccolo Allievi ${ }^{1}$, Paolo Goffredo ${ }^{2}$, Alan F. Utria ${ }^{2}$, Michele Pisano ${ }^{1}$, Elia Poiasina ${ }^{1}$, Alessandro Lucianetti ${ }^{1}$, \\ Paige Zhou', Imran Hassan ${ }^{2}$ \\ ${ }^{1}$ Department of Surgery, Papa Giovanni XXIII Hospital, Piazza OMS 1, Bergamo, Italy; ${ }^{2}$ Department of Surgery, University of Iowa Hospitals \& \\ Clinics, 200 Hawkins Dr, Iowa City, IA, USA \\ Contributions: (I) Conception and design: N Allievi, P Goffredo, I Hassan; (II) Administrative support: N Allievi, P Goffredo, P Zhou, I Hassan; (III) \\ Provision of study materials or patients: N Allievi, P Goffredo; (IV) Collection and assembly of data: N Allievi, P Goffredo, M Pisano; (V) Data \\ analysis and interpretation: All authors; (VI) Manuscript writing: All authors; (VII) Final approval of manuscript: All authors. \\ Correspondence to: Imran Hassan, MD, FACS, FASCRS. Department of Surgery, University of Iowa, 200 Hawkins Drive, 1516 JCP, Iowa City, IA \\ 52242, USA. Email: ihassan@mercycare.org.
}

Background: Recent evidence suggests that a mutation in the $K R A S$ gene has a significant impact on the clinical behavior and prognosis of patients with metastatic colorectal cancer. The $K R A S$ mutation $(m-K R A S)$ has been associated with decreased survival among patient undergoing treatment with a curative and palliative intent. This is believed to be secondary to a reduced response to anti-EGFR chemotherapy agents and a more intrinsically aggressive biology. The aims of this study were to identify risk factors for $m-K R A S$ in patients with curatively resected colorectal cancer and synchronous liver metastases and to assess its association with disease-specific survival (DSS).

Methods: The Surveillance, Epidemiology and End Results (SEER) Database was surveyed for patients undergoing resection of colorectal cancer and synchronous liver metastases from 2010 to 2015.

Results: A total of 806 patients were included, of which $40 \%$ had $m$-KRAS. Right-sided primary lesions (OR 2.56, 95\% CI: 1.90-3.44, P<0.001) and African-American ethnicity (OR 1.58, 95\% CI: 1.05-2.40, P=0.03) were independently associated with $m-K R A S$ on multivariable analysis. Compared to wild-type KRAS (wt-KRAS), m-KRAS was associated with decreased 3- and 5-year DSS (59\% vs. $50 \%$ and $29 \%$ vs. 21\%, respectively, $\mathrm{P}=0.024)$. After adjusting for confounders, a decreased DSS was observed in patients with rightsided lesions (HR 1.68, 95\% CI: 1.32-2.12, $\mathrm{P}<0.001$ ), while $m-K R A S$ was associated with a trend toward decreased DSS (HR 1.15, 95\% CI: 0.91-1.46, P=0.24).

Conclusions: In patients undergoing surgical resection of colorectal cancer and synchronous liver metastases, $m-K R A S$ was associated with right-sided lesions and African-American ethnicity. Compared to $w t-K R A S, m-K R A S$ was associated with a reduced DSS. Additionally, right-sided lesions were an independent negative prognostic factor for DSS.

Keywords: Colorectal cancer; KRAS; liver metastases

Submitted Jul 04, 2019. Accepted for publication Aug 01, 2019.

doi: $10.21037 /$ cco.2019.08.10

View this article at: http://dx.doi.org/10.21037/cco.2019.08.10 


\section{Introduction}

Colorectal cancer has an annual global incidence of 1.65 million cases (1). At the time of diagnosis, up to $25 \%$ of patients already have liver metastases and an additional $25 \%$ will develop hepatic metastases during the course of their disease (2). The median overall survival (OS) for patients with metastatic colorectal cancer that is treated with a palliative intent with multi-agent chemotherapy is approximately 30 months (3). Although surgical indications and timing of resections in colorectal cancer and liver metastases are still a matter of debate, hepatic metastasectomy with resection of the primary tumor remains the only potentially curative treatment (3-5). Mutations of the Kirsten Rat Sarcoma viral antigen homolog (KRAS) gene are found in about $40 \%$ of patients (6) with metastatic colorectal cancer. The determination of KRAS status is a relevant prognostic characteristic in these patients, as mutated KRAS (m-KRAS) reflects a poor response to anti-EGFR immunotherapy (3) and is associated with an increased cumulative incidence of metastatic disease as well as an adverse prognosis (6-8).

In recent years, the molecular pathways of oncogenesis in colorectal cancer and the intrinsic differences between rightsided and left-sided lesions have been investigated. Two metaanalyses $(6,9)$ of institutional studies evaluating the prognostic significance of $m-K R A S$ in patients treated with surgical resection of primary colorectal cancer and synchronous hepatic metastases, observed that $m-K R A S$ was associated with decreased OS and disease-free survival (DFS). More recently, a study (10) using the National Cancer Database (NCDB) confirmed the negative prognostic impact of $m-K R A S$ in colorectal cancer with synchronous liver metastases and demonstrated that $m-K R A S$ was associated with right-sided lesions and African-American ethnicity. Two main limitations of the NCDB are the lack of data on disease-specific survival (DSS) and the limited generalizability to the United States (U.S.) population as this database only collects data from the Commission on Cancer (CoC) affiliated Hospitals.

Therefore, aim of this study was to analyze the impact of $m-K R A S$ on DSS as well as its associated risk factors in patients undergoing resection of colorectal cancer and synchronous liver metastases in a U.S. population-based cohort utilizing the Surveillance, Epidemiology and End Results (SEER) database.

\section{Methods}

The SEER Program (11) provides information on cancer statistics within the U.S. It is supported by the Surveillance Research Program (SRP) within the National Cancer Institute (NCI). Data were available for a total of 18 registries representing approximately $30 \%$ of the U.S. population. The population covered by SEER is representative of the general U.S. population with regards to measures of income and education level. However, the SEER population presents a higher proportion of foreignborn patients as compared to the general U.S. population. SEER employs the International Classification of Diseases for Oncology, third edition (ICD-O-3) for histology classification (12).

In order to select patients diagnosed with colorectal cancer and synchronous liver metastases between 2010 and 2015, the Incidence-SEER 18 Regs Custom Data Colon and Rectum Database was queried (13). The selection of patients was conducted as follows: age older than 18 years, Stage IV colorectal adenocarcinoma with isolated liver metastases, resection of the primary tumor, nonprimary surgical procedure to distant site (i.e., patients undergoing liver resection) and known mutational status of KRAS.

Demographic variables included in the analyses were gender, age at diagnosis, year of diagnosis, ethnicity and insurance status. Clinicopathologic variables included administration of chemotherapy and radiation therapy, surgical treatment of the primary tumor and metastatic lesions, location and size of the primary tumor, involvement of the lymph nodes and KRAS status of the primary colorectal cancer. Primary tumor location was classified as 'right-sided' for lesions located from the cecum to the transverse colon and as 'left-sided' for lesions located from the splenic flexure to the rectum.

\section{Statistical analyses}

Descriptive statistics of demographic and clinicopathologic variables were performed. Student's $t$-test and Chi-square test were performed to compare continuous and discrete variables, respectively. Univariate and multivariate binary logistic regression was used to recognize factors associated with $m-K R A S$ and odds ratios (OR) and $95 \%$ CI were calculated. DSS was estimated with the Kaplan-Meier method and the log-rank test was used to evaluate statistical significance of the differences in survival. Cox proportional hazard regression was used for the multivariable survival model. Hazard ratios (HR) and 95\% confidence intervals were computed for the power of association between each variable and survival. 
Table 1 Characteristics of patients based on KRAS mutational status

\begin{tabular}{|c|c|c|c|}
\hline Patient variable & \multicolumn{2}{|c|}{ KRAS status } & $\mathrm{P}$ \\
\hline Sex (female), \% & 42.7 & 46.7 & 0.257 \\
\hline Age at diagnosis (years), mean (SD) & $57.3(13.7)$ & $59.4(13.1)$ & 0.038 \\
\hline Ethnicity, \% & & & 0.01 \\
\hline African-American & 11.3 & 19.0 & \\
\hline Other & 10.3 & 9.3 & \\
\hline Year of diagnosis, \% & & & 0.424 \\
\hline 2011 & 58 & 42 & \\
\hline 2015 & 57 & 43 & \\
\hline Insurance status, $\%$ & & & 0.368 \\
\hline Uninsured & 5.4 & 4.7 & \\
\hline Medicaid & 11.5 & 11.8 & \\
\hline Insured & 82.1 & 72.5 & \\
\hline Unknown & 1 & 11 & \\
\hline Radiotherapy (received), \% & 9.9 & 11.8 & 0.639 \\
\hline
\end{tabular}

Data analyses were performed using SEER*Stat software (14) and Statistical Package for the Social Sciences (SPSS) software (version 20.0; SPSS Inc., Chicago, IL, USA). $P$ values were considered significant if $<0.05$ and all tests were two-sided. Considering that data included in the SEER Database is publicly available and de-identified, approval by an institutional review board was not considered necessary for the current study.

\section{Results}

\section{Patient characteristics}

A total number of 806 patients with colorectal cancer and synchronous liver metastases and documented KRAS mutational status who underwent surgical treatment of their primary tumor and liver metastases were retrieved in the SEER Database. $m-K R A S$ was present in 321 cases (39.8\%). Overall $m$-KRAS was associated with older age (59.4 vs. 57.3 years, $\mathrm{P}=0.038$; Table 1) and AfricanAmerican ethnicity $(19.0 \%$ vs. $11.3 \%, \mathrm{P}=0.01)$, but was not related to gender, insurance status or year of diagnosis. Furthermore, $m-K R A S$ was more commonly found in rightsided primary lesions, when compared to left-sided lesions (54.7\% vs. $31.3 \%, \mathrm{P}<0.001$ ). Clinicopathologic variables, such as primary tumor size, lymph node involvement and radio-chemotherapy administration were not significantly associated with $m-K R A S$. On multivariable analysis, rightsided lesions (OR 2.56, 95\% CI: 1.90-3.44, $\mathrm{P}<0001$ ) and African-American ethnicity (OR 1.58, 95\% CI: 1.05-2.40, 
Table 2 Multivariable analysis for predictors of $m-K R A S$ status

\begin{tabular}{lccc}
\hline Patient variable & OR & $95 \% \mathrm{Cl}$ & $\mathrm{P}$ \\
\hline Ethnicity & & & \\
Caucasian & Reference & - & - \\
African-American & 1.58 & $1.05-2.40$ & 0.03 \\
Other & 1.08 & $0.66-1.77$ & 0.77 \\
Primary tumor location & & & \\
Left-sided & Reference & - & - \\
Right-sided & 2.56 & $1.90-3.44$ & $<0.001$ \\
\hline
\end{tabular}

Age and gender were not significantly associated with $m-K R A S$. OR, odds ratio; $\mathrm{Cl}$, confidence interval.

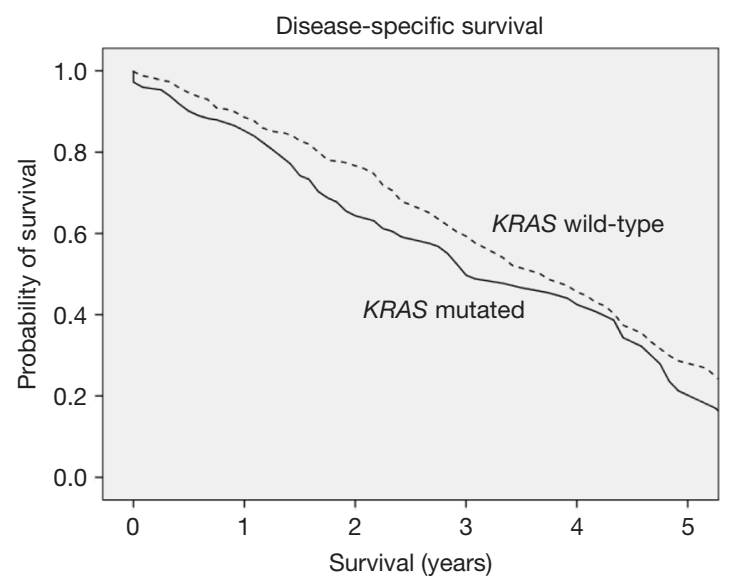

Figure 1 DSS by KRAS mutational status. Log-rank test: $\mathrm{P}=0.024$. DSS, disease-specific survival.

Table 3 Multivariable analysis for prognostic factors

\begin{tabular}{lccc}
\hline Patient variable & HR & $95 \% \mathrm{Cl}$ & $\mathrm{P}$ \\
\hline Right-sided primary lesion & 1.68 & $1.32-2.12$ & $<0.001$ \\
$m-K R A S$ & 1.15 & $0.91-1.46$ & 0.24
\end{tabular}

Age, gender, ethnicity, lymph node involvement and primary tumor size were not significant. $\mathrm{HR}$, hazard ratio; $\mathrm{Cl}$, confidence interval.

$\mathrm{P}=0.03)$ were significantly associated with $m$-KRAS status (Table 2).

\section{Survival analyses}

Compared to $w t-K R A S, m-K R A S$ status was associated with decreased DSS; 3 - and 5 -year DSS were $59 \%$ vs. 50\%, and $29 \%$ vs. $21 \%$, respectively ( $\mathrm{P}=0.024$; Figure 1). Similarly, patients with right-sided lesions demonstrated an increase in disease-specific mortality, with a 5 -year DSS of $19 \%$, as compared to $29 \%$ for left-sided lesions $(\mathrm{P}<0.001)$.

After adjusting for the available confounders, patients with right-sided lesions had a decreased DSS (HR 1.68, 95\% CI: 1.32-2.12, $\mathrm{P}<0.001$; Table 3). Furthermore, $m$-KRAS demonstrated a trend toward decreased DSS (HR 1.15, 95\% CI: 0.91-1.46, $\mathrm{P}=0.24)$.

\section{Discussion}

The present study investigated the associations between KRAS mutational status, primary tumor location and survival in a cohort of 806 patients from a population-based dataset. African-American ethnicity and right-sided lesions were independently associated with $m-K R A S$. Moreover, $m-K R A S$ and the presence of a right-sided primary lesion were negative prognostic factors in patients with colorectal cancer and synchronous liver metastases undergoing surgical resection of both their primary colorectal cancer and liver metastases.

Given its relatively high incidence, the relative ease of detectability, and the various targeted therapeutic options available $(3,4), m-K R A S$ is an important prognostic consideration in the management of metastatic colorectal cancer. In the context of multi-disciplinary strategies, the decision-making process is often driven by clinical and radiological features, rather than pathologic and biologic elements. However, the mutational status of KRAS may predict the responsiveness to upfront chemotherapy in presence colorectal cancer and synchronous liver metastases (15) and may also explain intrinsic aggressive tumor biology $(16,17)$, resulting in increased risk of progression and relapse after resection. $m$-KRAS is acquired early in the mutational cascade, it remains stable over the course of the disease, and has a high concordance between primary tumor and liver metastases (18). Several preoperative clinical risk scores for patients presenting with resectable colorectal cancer and synchronous liver metastases were developed (19-22) based on institutional cohorts, although there have been concerns regarding the absence of external validation of these scores as well as the accuracy and applicability of the reported prognostic factors (23). Therefore, using current knowledge of the mutational profile of colorectal cancer to guide clinical practice could harbor the potential of improving oncologic 
outcomes. This has led to the creation of the Genetic and Morphological Evaluation (GAME) Score (24), which is based on morphologic and biologic tumor information and represents the first clinical risk score including the genetic status $(K R A S)$ of the primary tumor. The score was developed using a derivation cohort, including 502 patients, and an external validation cohort (747 patients) and its discriminatory capacity resulted to be superior to other institutionally derived scores. Integrating genetic, biologic and clinical information not only is likely to improve comparison of different cohorts, but may also guide treatment selection and provide relevant prognostic details (24).

The proportion of patients with $m-K R A S$ in the current study (39.8\%) was consistent with previous reports in the literature (14-46\%). In an analysis of all Stage IV colorectal cancers within the SEER database, Charlton et al. (25) reported an overall mutation rate of 44\% among 6794 patients. Similarly, in their study utilizing the NCDB, Goffredo et al. (10) reported $m-K R A S$ in $42 \%$ out of 2,655 patients who underwent surgical treatment of both their primary colorectal cancer and isolated liver metastases. Two meta-analyses of institutional studies by Brudvik et al., that included 14 studies with 1,809 cases, and Tosi et al., which included 11 studies with 1,369 patients, reported an overall $m$-KRAS incidence of $30.6 \%$ and $34.3 \%$, respectively. While there was substantial overlap between these two studies, they differed in that Tosi et al. used a more stringent criteria for inclusion in their analysis and added three newer studies $(6,9)$. Both these meta-analyses demonstrated that $m-K R A S$ was negatively associated with OS and relapse free survival, irrespective of chemotherapy agent received.

In our cohort, the proportion of patients with a rightsided cancer within the $m$-KRAS subgroup was high at $54 \%$, as compared to $31 \%$ within the wt-KRAS subgroup. In a meta-analysis including 66 studies and 1,437,846 patients, Petrelli et al. (26) reported that right-sided lesions were associated with worse OS. There have been several explanations in the literature for this difference in survival based on location. Firstly, surgical approaches to rightsided and left- sided colon/rectal cancer are different: while the standard of care is well defined for left-sided and rectal lesions, the optimal surgical resection for right-sided lesions remains debated, particularly in regards to the extent of mesocolic excision and lymphadenectomy $(27,28)$. Secondly, benefits from anti-EGFR agents is less pronounced in right-sided lesions, as reported by two recent trials $(29,30)$.
Finally, right-sided colon cancer is associated with increased incidence of genetic mutations [microsatellite instability (MSI), BRAF and KRAS mutations] which may explain the survival difference in this subgroup of patients (31).

In the current study, right-sided tumors and AfricanAmerican ethnicity were significantly associated with the presence of $m-K R A S$ status. Right-sided lesions, but not African-American ethnicity, were associated with lower DSS on multivariable survival analysis. Using the NCDB, Goffredo et al. found comparable results in a U.S. based national cohort of patients (10), even though the NCDB and SEER databases are different in terms of quality and origin of data. The former collects data from Commission on Cancer-accredited cancer program registries, capturing around $70 \%$ of all newly diagnosed cases of cancer at an institutional level; the latter gathers data from populationbased cancer registries and covers $34.6 \%$ of the U.S. population. Notwithstanding the possible overlapping between the two datasets, the reproducibility of results between these two studies underscores the validity of the association between $m$-KRAS and survival outcomes. Our findings appear to support the hypothesis that specific tumor biology and mutational status may be related to primary tumor location and could be the main driver for worse outcomes.

The present study has several limitations. Firstly, the retrospective nature of the analysis of the SEER database might be flawed by intrinsic biases of large databases. Secondly, the SEER is a population-based dataset and several potentially significant variables are not collected routinely. Thirdly, the vast range of therapeutic possibilities, including type of surgical resection, various lines of chemotherapy, radiotherapy and their temporal combination, could not be investigated in depth. Finally, the accuracy and extent of surgical treatment as well as other therapeutic maneuvers could not be evaluated.

\section{Conclusions}

In conclusion, the present study suggests a negative prognostic value of $m-K R A S$ and right-sided lesions in a population-based cohort of patients undergoing resection of colorectal cancer and synchronous liver metastases. Our data show that African-American ethnicity and right-sided lesions were associated with $m$-KRAS. The mutational status of KRAS is likely to be associated with unfavorable tumor biology leading to decreased survival and should be discussed in the context of multi-disciplinary management 
of patients with colorectal cancer and synchronous liver metastases.

\section{Acknowledgments}

None.

\section{Footnote}

Conflicts of Interest: The authors have no conflicts of interest to declare.

Ethical Statement: The authors are accountable for all aspects of the work in ensuring that questions related to the accuracy or integrity of any part of the work are appropriately investigated and resolved. The data included in the SEER Database is publicly available and deidentified, approval by an institutional review board was not considered necessary for the current study.

\section{References}

1. Global Burden of Disease Cancer Collaboration, Fitzmaurice C, Allen C, Barber RM, et al. Global, Regional, and National Cancer Incidence, Mortality, Years of Life Lost, Years Lived With Disability, and DisabilityAdjusted Life-years for 32 Cancer Groups, 1990 to 2015: A Systematic Analysis for the Global Burden of Disease Study. JAMA Oncol 2017;3:524-48.

2. van der Pool AE, Damhuis RA, Ijzermans JN, et al. Trends in incidence, treatment and survival of patients with stage IV colorectal cancer: a population-based series. Colorectal Dis 2012;14:56-61.

3. Van Cutsem E, Cervantes A, Adam R, et al. ESMO consensus guidelines for the management of patients with metastatic colorectal cancer. Ann Oncol 2016;27:1386-422.

4. Adam R, Aimery de Gramont A, Joan Figueras AG et al. The Oncosurgery Approach to Managing Liver Metastases from Colorectal Cancer: A Multidisciplinary International Consensus. Oncologist 2012;17:1225-39.

5. Chow FCL, Chok KSH. Colorectal liver metastases: An update on multidisciplinary approach. World J Hepatol 2019;11:150-72.

6. Tosi F, Magni E, Amatu A, et al. Effect of KRAS and BRAF Mutations on Survival of Metastatic Colorectal Cancer After Liver Resection: A Systematic Review and Meta-Analysis. Clin Colorectal Cancer 2017;16:e153-63.

7. Kim MJ, Lee HS, Kim JH, et al. Different metastatic pattern according to the KRAS mutational status and site-specific discordance of KRAS status in patients with colorectal cancer. BMC Cancer 2012;12:347.

8. Cejas P, López-Gómez M, Aguayo C, et al. KRAS mutations in primary colorectal cancer tumors and related metastases: A potential role in prediction of lung metastasis. PLoS One 2009;4:e8199.

9. Brudvik KW, Kopetz SE, Li L, et al. Meta-analysis of KRAS mutations and survival after resection of colorectal liver metastases. Br J Surg 2015;102:1175-83.

10. Goffredo P, Utria AF, Beck AC, et al. The Prognostic Impact of KRAS Mutation in Patients Having Curative Resection of Synchronous Colorectal Liver Metastases. J Gastrointest Surg 2019;23:1957-63.

11. Available online: https://seer.cancer.gov/

12. Fritz A, Jack A, Shanmugarathnam K, et al. International Classification of Disease for Oncology. 3rd ed. Geneva: World Health Organization, 2000.

13. Surveillance, Epidemiology, and End Results (SEER) Program. SEER*Stat Database: Incidence - SEER 18 Regs Custom Data Colon and Rectum (with SSF 9 - in-house and treatment fields. Available online: www.seer.cancer.gov

14. Surveillance Research Program, National Cancer Institute SEER*Stat software (version 8.3.5). Available online: www. seer.cancer.gov/seerstat

15. Zimmitti G, Shindoh J, Mise Y, et al. RAS Mutations Predict Radiologic and Pathologic Response in Patients Treated with Chemotherapy Before Resection of Colorectal Liver Metastases. Ann Surg Oncol 2015;22:834-42.

16. Italiano A, Hostein I, Soubeyran I, et al. KRAS and BRAF Mutational Status in Primary Colorectal Tumors and Related Metastatic Sites: Biological and Clinical Implications. Ann Surg Oncol 2010;17:1429-34.

17. Margonis GA, Kim Y, Spolverato G, et al. Association between specific mutations in KRAS codon 12 and colorectal liver metastasis. JAMA Surg 2015;150:722-9.

18. Knijn N, Mekenkamp LJM, Klomp M, et al. KRAS mutation analysis: a comparison between primary tumours and matched liver metastases in 305 colorectal cancer patients. Br J Cancer 2011;104:1020-6.

19. Fong Y, Fortner J, Sun RL, et al. Clinical Score for Predicting Recurrence After Hepatic Resection for Metastatic Colorectal Cancer. Ann Surg 1999;230:309.

20. Iwatsuki S, Dvorchik I, Madariaga JR, et al. Hepatic resection for metastatic colorectal adenocarcinoma: a proposal of a prognostic scoring system. J Am Coll Surg 1999;189:291-9. 
21. Nordlinger B, Guiguet M, Vaillant JC, et al. Surgical resection of colorectal carcinoma metastases to the liver. A prognostic scoring system to improve case selection, based on 1568 patients. Association Française de Chirurgie. Cancer 1996;77:1254-62.

22. Rees M, Tekkis PP, Welsh FKS, et al. Evaluation of Long-term Survival After Hepatic Resection for Metastatic Colorectal Cancer. Ann Surg 2008;247:125-35.

23. Zakaria S, Donohue JH, Que FG, et al. Hepatic resection for colorectal metastases: value for risk scoring systems? Ann Surg 2007;246:183-91.

24. Margonis GA, Sasaki K, Gholami S, et al. Genetic And Morphological Evaluation (GAME) score for patients with colorectal liver metastases. Br J Surg 2018;105:1210-20.

25. Charlton ME, Kahl AR, Greenbaum AA, et al. KRAS Testing, Tumor Location, and Survival in Patients With Stage IV Colorectal Cancer: SEER 2010-2013. J Natl Compr Canc Netw 2017;15:1484-93.

26. Petrelli F, Tomasello G, Borgonovo K, et al. Prognostic survival associated with left-sided vs right-sided colon cancer a systematic review and meta-analysis. JAMA Oncol 2017;3:211-9.

27. Vogel JD, Eskicioglu C, Weiser MR, et al. The American

Cite this article as: Allievi N, Goffredo P, Utria AF, Pisano M, Poiasina E, Lucianetti A, Zhou P, Hassan I. The association of $K R A S$ mutation with primary tumor location and survival in patients undergoing resection of colorectal cancers and synchronous liver metastases. Chin Clin Oncol 2019;8(5):46. doi:10.21037/cco.2019.08.10 society of colon and rectal surgeons clinical practice guidelines for the treatment of colon cancer. Dis Colon Rectum 2017;60:999-1017.

28. Bertelsen CA, Neuenschwander AU, Jansen JE, et al. Disease-free survival after complete mesocolic excision compared with conventional colon cancer surgery: a retrospective, population-based study. Lancet Oncol 2015;16:161-8.

29. Venook AP, Niedzwiecki D, Innocenti F, et al. Impact of primary (1o) tumor location on overall survival (OS) and progression-free survival (PFS) in patients (pts) with metastatic colorectal cancer (mCRC): Analysis of CALGB/ SWOG 80405 (Alliance). J Clin Oncol 2016;34:3504.

30. Heinemann V, Modest DP, Fischer von Weikersthal L, et al. Gender and tumor location as predictors for efficacy: Influence on endpoints in first-line treatment with FOLFIRI in combination with cetuximab or bevacizumab in the AIO KRK 0306 (FIRE3) trial. J Clin Oncol 2014;32:3600.

31. Chiu JW, Krzyzanowska MK, Serra S, et al. Molecular Profiling of Patients With Advanced Colorectal Cancer: Princess Margaret Cancer Centre Experience. Clin Colorectal Cancer 2018;17:73-9. 